\title{
Association between haptoglobin genotype polymorphism and type two (2) diabetes in Accra, Ghana
}

\author{
Charles Brown ${ }^{1,}$, Benedicta Awisi ${ }^{1}$, Harry Asmah ${ }^{1}$, Batholomew Dzudzor ${ }^{2}$, Anita Ghansah ${ }^{3}$ \\ ${ }^{1}$ Medical Laboratory Sciences Department, School of Allied Health Sciences, University of Ghana, Korle -bu - Accra, Ghana \\ ${ }^{2}$ University of Ghana Medical School, University of Ghana, Korle -bu - Accra, Ghana \\ ${ }^{3}$ Noguchi Memorial Institute for Medical Research, University of Ghana, Legon - Accra, Ghana
}

\section{Email address:}

cabrown@chs.edu.gh (C. Brown)

\section{To cite this article:}

Charles Brown, Benedicta Awisi, Harry Asmah, Batholomew Dzudzor, Anita Ghansah. Association between Haptoglobin Genotype Polymorphism and Type Two (2) Diabetes in Accra, Ghana. American Journal of Biomedical and Life Sciences. Vol. 1, No. 4, 2013, pp. 103-109. doi: 10.11648/j.ajbls.20130104.15

\begin{abstract}
Polymorphism of the haptoglobin (Hp) gene, characterized by alleles $\mathrm{Hp} 1$ and $\mathrm{Hp} 2$, gives rise to structurally and functionally distinct Hp protein phenotypes: Hp1-1, Hp2-1, and Hp2-2. The corresponding proteins have structural and functional differences that have influence on a particular disease. For example, Hp genotype is an independent risk factor for diabetic complications. In urban Ghana, type two diabetes mellitus (T2DM) affects at least $6 \%$ of adults. The aim of this study was to assess the association between Hp genotype polymorphism in T2DM patients in Accra. The study was a case control one. A total of 100 participants, 50 T2DM patients attending the Diabetes Clinic (Korle-Bu Teaching Hospital) and 50 healthy non-diabetic controls, were involved. Plasma glucose concentration was measured by the glucose-oxidase method. Fasting blood glucose was performed on all subjects except for the individuals with a history of T2DM. Hp genotype was determined by allele specific polymerase chain reaction (PCR). PCR produced Hp genotype-specific bands for the Hp1F, $\mathrm{Hp} 1 \mathrm{~S}$, and Hp2 alleles. Statistical analyses revealed a significant difference in the Hp genotype distribution between diabetics and non-diabetics $\left(\chi^{2}=7.84, \mathrm{df}=2, p=0.0198\right)$. Hp1-1 was the most frequent genotype among non-diabetics $(58 \%)$ whilst Hp2-2 (38\%) was most frequent genotype among diabetics. Majority of the diabetics were found in the Hp1S-1F and Hp2-2 genotype groups for diastolic BP $(\mathrm{mmHg})$, systolic BP $(\mathrm{mmHg})$ and FBG $(\mathrm{mM})$. There was a strong association between DM and Hp2-2 genotype, followed by Hp2-1 (Hp1F-2 > Hp1S- 2) with the least being Hp1-1 (Hp1F-1F, Hp1S-1F, Hp1S-1S). The risk of developing diabetes among people with Hp2-2 and Hp1F-2 genotypes was high. They can therefore be used as markers for an individual developing DM.
\end{abstract}

Keywords: Haptoglobin, Genotype, Polymorphism, Type two (2) Diabetes

\section{Introduction}

Haptoglobin (Hp) is a plasma protein that owes its name to its hemoglobin $(\mathrm{Hb})$-binding properties [1]. These properties have led to its description as an antioxidant protein because binding to free $\mathrm{Hb}$ inhibits $\mathrm{Hb}$-induced oxidative tissue damage. Haptoglobin is mainly synthesized by hepatocytes, its levels increasing during inflammation or infection. In humans, two alleles for $\mathrm{Hp}$ have been described that give rise to different $\mathrm{Hp}$ proteins and three major phenotypes, Hpl-1 (homozygous for the 1 allele), Hp2-2 (homozygous for the 2 allele) or Hp2-1 (the heterozygote) [2]. Hp types exhibit some differences in function: Hp 1-1 has a strong capacity for hemoglobin binding and anti-oxidative function, $\mathrm{Hp} 2-2$ functions weakly in hemoglobin binding and anti-oxidative capacity, and Hp2-1 is a functional intermediate [3].

In both type 1 (T1) and type 2 (T2) diabetes mellitus (DM), complications in target organs arise from oxidative stress resulting primarily from chronic hyperglycemia [4]. The generation of this oxidative stress may be moderated by exogenous and host genetic factors that scavenge free radicals. Endogenous antioxidants such as glutathione and certain Hp phenotypes are important in the prevention and protection of blood vessel walls of internal organs against oxidative stress which is normally increased in the diabetic state by the presence of glycated $\mathrm{Hb}$ [5]. Extravascular and intravascular haemolysis occur at higher rates in DM individuals compared to non-DM individuals, thus increasing the amount of $\mathrm{Hp}-\mathrm{Hb}$ molecules in plasma and tissues [6]. 
There are functional differences among the various Hp phenotypes [7]. These functional differences invariably associate Hp polymorphism with the prevalence and clinical evolution of many autoimmune and inflammatory diseases. The Hp polymorphism arises from variant $\alpha$-chains [8].

Inherited factors play an important role in the pathogenesis of T2DM [9]. The increased oxidative stress in diabetic patients results in the oxidation of glucose and the modification of low-density lipoproteins (LDL). These changes may stimulate the production of inflammatory cytokines that have been implicated in the morphological and pathological changes found in macro vascular and micro vascular complications.

A study by Adinortey and associates in 2009 [10] reported of an association between diabetic complications and $\mathrm{Hp}$ and diabetic retinopathy among Ghanaians. Hp 2-2 is known to be associated with diabetic vascular complications such as neuropathy, nephropathy and hypertension [3]. There is paucity of information on the role of genetic factors, especially those relating to $\mathrm{Hp}$ genotype in the expression of complications among T2DM patients in Ghana. Therefore there is the need to find out the association between Hp genotype polymorphism and T2DM. The aim of this study was to assess the association between Hp genotype polymorphism and its occurrence in T2DM individuals in Accra.

\section{Materials and Methods}

\subsection{Study Design and Study Participants}

The study was a case control one. Patients were selected from the Diabetes Clinic of the Korle- $\mathrm{Bu}$ Teaching Hospital (KBTH), Accra, Ghana. The Clinic serves as the Center for National Diabetes Management and Research. Patients assemble here before their appointment times for a health education talk which details basic foot care, blood pressure regulation, diet and physical activity. Their blood glucose levels and blood pressures are also assessed.

\subsubsection{Inclusion and Exclusion Criteria}

Confirmed T2DM patients with or without complications and newly diagnosed T2DM patients (fasting plasma venous glucose of $\geqslant 7 \mathrm{mmol} / \mathrm{l}$ or random glucose test $>11 \mathrm{mmol} / 10$ ) [11] attending the Diabetic Clinic were included. Non-diabetics (those with no history of diagnosis of diabetes, fasting plasma glucose $<5.6$ and $2 \mathrm{hr}$ plasma glucose $<7.8 \mathrm{mM}$ for oral glucose tolerance test - WHO criterion) served as controls. T1DM patients and pregnant women were excluded.

\subsection{Ethics}

Ethical clearance was obtained from the Ethics and Protocol Review Committee of the School of Allied Health Sciences, University of Ghana, Korle-bu. In addition, each participant provided written informed consent and completed a questionnaire.

\subsection{Blood Sample Collection}

Overnight fasting blood sample volumes of $3 \mathrm{ml}$ was collected from each participant into fluoride tubes for glucose determination and EDTA tubes for DNA extraction. The tubes were centrifuged at 2500rpm for 3 min (fluoride tubes) and at 4500rpm for 5 min (EDTA tubes) using a Hitachi 20PR-52D centrifuge. The buffy coat from the EDTA tube was collected into Eppendorf tubes and stored at $-20^{\circ} \mathrm{C}$. Clinical measurement was undertaken using the supernatant from the fluoride tubes.

\subsection{Clinical Assessment}

The clinical history of all participants, including demographic data, disease condition, treatment history and family history of diabetes, was taken. Each participant's BMI was calculated by taking the height using a stadiometer and the weight using a weighing scale. The body mass index BMI was calculated as weight in kilograms divided by the square of height in meters $(\mathrm{kg} / \mathrm{m} 2)$.

With the use of a sphygmomanometer the blood pressure (BP) of each participant was measured after 5 minutes of rest in a sitting position [12].

\subsection{Glucose Estimation}

The concentration of plasma glucose was estimated using an A25 BioSystems (Madrid, Spain) at the Chemical Pathology Laboratory of the School of Allied Health Sciences, Korle- Bu. The estimation was done using a commercial kit (Biosystem S.A, Costa Brava, Spain).

\subsection{DNA Extraction}

DNA was extracted from the blood samples using the QIAamp Mini Kit Tissue Protocol (Qiagen Inc., Valencia, USA) following the manufacturer's instructions. DNA was divided into two aliquots of $30 \mu \mathrm{l}$ each and stored at $-20^{\circ} \mathrm{C}$.

\subsection{Haptoglobin Genotyping}

Hp genotype was determined by allele specific polymerase chain reaction as described [13].

Three PCRs were performed on each sample to genotype the Hp1S, 1F, and 2 alleles using forward primers C51, 5'-GCA ATG ATG TCA CGG ATATC-3'and F3, 5'-CAG GAG TAT ACA CCT TAA ATG-3' and reverse primers C42, 5'-TTA CAC TGG TAG CGA ACC GA-3', C72, 5'-AAT TTA AAA TTG GCAT TT CGCC-3'and S2, 5'-TTA TCC ACT GCT TCT CAT TG-3'. The combination of F3 and C42 identified the $\mathrm{Hp} 2$ allele, $\mathrm{C} 51$ and S2 primers the Hp1S allele, and F3 and C72 primers the Hp1F allele. The PCR was performed using 0.25 units of Taq DNA polymerase $(5 \mathrm{u} / \mu \mathrm{l})$ (Sigma Missouri, USA), with $3 \mu \mathrm{l}$ of $10 \times$ PCR buffer (with $\mathrm{MgCl}_{2}$ ), oligonucleotide primers $(1.0 \mu \mathrm{l}$ each) at $10 \mu \mathrm{M}$ and dNTPs $(0.5 \mu \mathrm{l}$ each) at $10 \mathrm{mM}$. Conditions for amplification were an initial denaturation at $94^{\circ} \mathrm{C}$ for 15 minutes, 35 cycles of denaturation at $94^{\circ} \mathrm{C}$ for 40 seconds, 
primer annealing at $52^{\circ} \mathrm{C}$ for 1 minute for the $\mathrm{C} 51-\mathrm{S} 2$ and $\mathrm{F} 3-\mathrm{C} 72$ primer reactions and $58^{\circ} \mathrm{C}$ for 1 minute for the F3-C42 primer reactions and strand extension by the Taq polymerase at $72^{\circ} \mathrm{C}$ for 2 minutes. A final strand extension at $72^{\circ} \mathrm{C}$ for 5 minutes was then performed to complete the reaction.

After the reaction $10 \mu \mathrm{l}$ of the PCR product was run by electrophoresis at 120 volts in $1 \%$ agarose gel (Biopioneer Co, USA) stained with $0.5 \mu \mathrm{g} / \mathrm{ml}$ ethidium bromide (Life Technologies Co, USA) in 1X Tris acetate EDTA (TAE) running buffer (Biopioneer Co, USA) using $2 \mu 1$ of blue/orange DNA loading dye (6X) (Promega Co, USA). A hundred base pair molecular size marker/ladder (Sigma Mo, USA) was run alongside the PCR products on the gel. The gel was photographed using UV-illumination (UVIsave gel documentation system, model GAS9200/1/2/3, version 12) and analyzed.

\subsection{Statistical Analysis}

Data was entered into Microsoft Excel 2010. Analyses were performed using Systat for Windows (version 13.00.05) and MedCalc (version 12.7.1.0). Hardy-Weinberg equilibrium was tested using the Chi-square goodness-of-fit test. Genotypic distributions in different groups were compared using the Chi-square test. Student's t-test and analysis of variance (ANOVA) were used to compare continuous variables expressed as means and standard deviations. ORs and 95\% confidence intervals (CIs) were calculated. All tests were two-tailed and $\mathrm{p}<0.05$ was considered statistically significant.

\section{Results}

\subsection{Study Population}

The study comprised of a 100 subjects, 50 T2DM individuals (12 males and 38 females) and 50 non diabetics (11 males and 39 females) [Table 1]. The male diabetics were significantly older than male non-diabetics $(\mathrm{p}<$ 0.0001 ), however there was no statistically significant difference between the ages of the female diabetics and female non-diabetics $(p>0.24)$.

\subsection{Clinical and Biochemical Indices of Non-diabetics and Diabetics}

Table 1 shows the clinical and biochemical indices of the participants. The BMI of diabetic and non-diabetic males showed no significant difference $(p=0.29)$ though that between the females was significant $(p=0.003)$. For the diabetics, the females had higher BMI values.

The mean systolic and diastolic BPs were significantly higher for the diabetics compared to the non-diabetics (all $\mathrm{p}$ $<0.05)$. Sex differences were observed. The mean systolic BP between diabetic and non-diabetic males was significant $(\mathrm{p}=0.04)$ whilst the mean diastolic BP between diabetic and non-diabetic females was significant $(\mathrm{p}=0.01)$.

The mean FBG for the diabetics was significantly higher than that of the non-diabetics (all $\mathrm{p}<0.0001$ ). The FBGs for the diabetic males and females were also significantly higher than that of their non-diabetic counterparts (all $\mathrm{p}<0.05$ ).

\subsection{Hp Genotyping Distribution}

PCR produced Hp genotype-specific bands: products of 1,400, 1,200, and 935 base pairs were obtained for the Hp1F (Fig. 1a), Hp1S (Fig. 1b), and $\mathrm{Hp} 2$ (Fig. 1c) alleles respectively. However, 11 diabetic and 17 non-diabetic individuals could not be genotyped.

Genotype frequencies were in Hardy-Weinberg equilibrium for diabetics $(\chi 2=1.93, \mathrm{p}=0.165)$ but not for non-diabetics $(\chi 2=11.52, \mathrm{p}=0.0007)$. For diabetics, the $\mathrm{Hp} 1$ allele frequency was 0.506 and that for $\mathrm{Hp} 2$ allele was 0.494; while for non-diabetics it was 0.603 and 0.397 for $\mathrm{Hp} 1$ and $\mathrm{Hp} 2$ alleles, respectively.

Statistical analyses revealed a significant difference in the Hp distribution between diabetic and non-diabetics $(\chi 2=$ 7.84 , df $=2, \mathrm{p}=0.0198)$. Hp1-1 was the most frequent genotype among non-diabetics (58\%) whilst Hp2-2 (38\%) was most frequent genotype among diabetics. Non diabetics outnumbered the diabetics in the Hp1F-1F group whereas diabetics outnumbered the non-diabetics in the Hp1S-1F, Hp2-1 and Hp2-2 groups (Fig. 2).
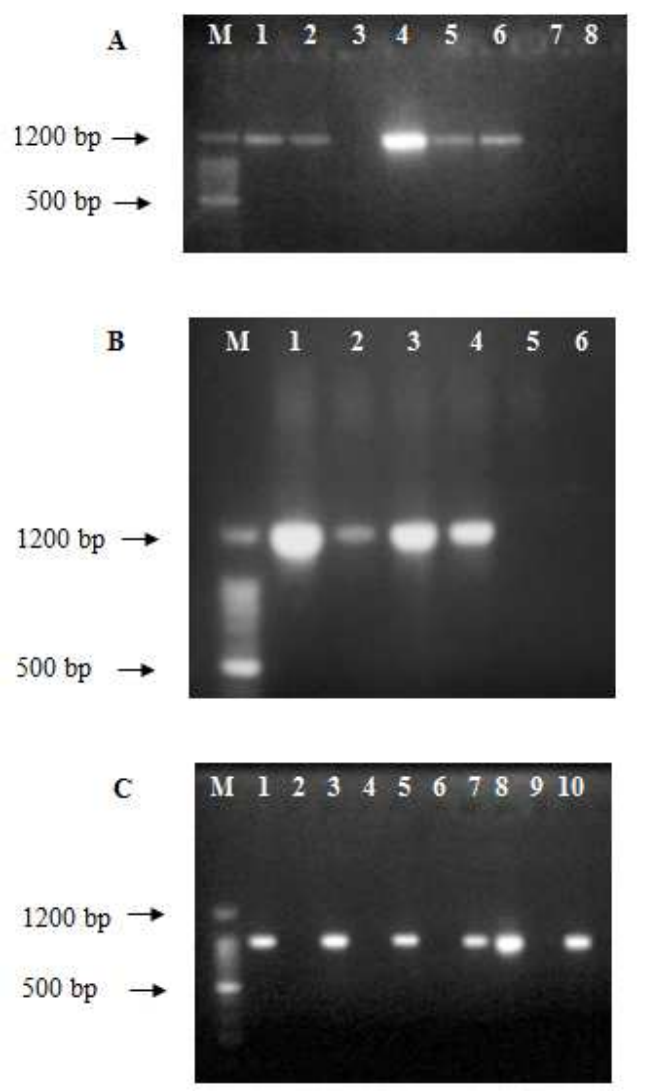

Figure 1. Examples of an ethidium bromide-stained 1.0\% agarose gel electrophoresis of PCR products for Hp genotyping (a) Lanes 1, 2 and 4-6 show PCR positives for HplF (b) Lanes 1-4 show PCR positives for HplS (c) Lanes 1, 3, 5, 7, 8 and 10 show PCR positives for Hp2. M=100 bp marker. 


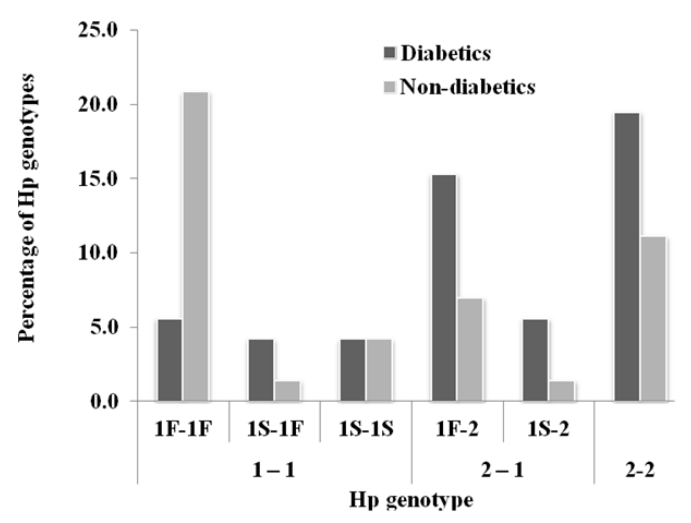

Figure 2. Percentage distribution of Hp genotypes among diabetics and non-diabetics.

Comparing the allele frequency among the diabetics and non-diabetics studied, a statistically significant difference was found between Hp1-1 and Hp2-1 (OR $=4.75,95 \%$ CI $1.41-16.05, \mathrm{p}=0.012), \mathrm{Hp} 1-1$ and Hp2-2 $(\mathrm{OR}=0.30,95 \%$ CI $0.09-0.96, \mathrm{p}=0.042$ ) but not between Hp2-1 and Hp2-2 $(\mathrm{OR}=0.7,95 \%$ CI $0.19-2.53, \mathrm{p}=0.59)$. Statistically significant differences (Fisher's exact test, two-tailed, all p's $<0.05$ ) were also found between $1 \mathrm{~F}-1 \mathrm{~F}$ and $1 \mathrm{~F}-2,1 \mathrm{~F}-1 \mathrm{~F}$ and $1 \mathrm{~S}-2$ and $1 \mathrm{~F}-1 \mathrm{~F}$ and $2-2$ among the diabetics and non-diabetics.

Dot density plots of $\mathrm{Hp}$ genotype distribution and diastolic BP (mmHg), systolic BP ( $\mathrm{mmHg})$ and FBG $(\mathrm{mM})$ for the diabetics and non-diabetics, Figs. $3 \mathrm{a}-\mathrm{c}$, show that majority of the diabetics are found in the Hp1F-2 and Hp2-2 genotype groups. On the other hand, for the non-diabetics, the majority are in the Hp1-1 genotype group.

\section{Discussion}

Diabetes has been projected to be the 7 th leading cause of death in 2030 [14]. Type 2 diabetes comprises $90 \%$ of people with diabetes around the world [11], and is largely the result of excess body weight and physical inactivity. Until recently, this type of diabetes was seen only in adults but it is now also occurring in children. Simple lifestyle measures have been shown to be effective in preventing or delaying the onset of type 2 diabetes. Early detection of the disease and timely interventions are important as they can help reduce the morbidity and mortality associated with it. Earlier studies have strongly linked DM to Hp polymorphisms $[15,16]$. However, these studies focused on the Hp phenotype. This study thus evaluated association of $\mathrm{Hp}$ genotype polymorphism and T2DM in subjects from Accra, Ghana.

In this study, diabetic patients recorded higher BMIs than the non-diabetics which is consistent with the observation that a high BMI predicts the development of several diseases, including T2 diabetes [17,18]. The female diabetics recorded the highest BMIs. This agrees with earlier work [19]. The high BMI puts the female diabetics at a higher risk of developing other risk factors for CVDs such as hypertension and high serum cholesterol.

Fasting blood glucose levels for the non - diabetic participants were within the reference range and were significantly lower compared to the levels for the T2DM participants. This confirms other reports $[15,18]$.

Haptoglobin gene polymorphism has been investigated in case-control and cross sectional studies of several diseases in relation to their incidence and clinical expression in particular disease of parasitic origin (malaria) [19], infectious disease (pulmonary tuberculosis) [20] and non-infectious disease (diabetes) [15,16]. There is controversy on whether there is any association between $\mathrm{Hp}$ phenotypes and predisposition to DM. For example, a report by Awadallah and Hamad [21] suggests that the prevalence of diabetes is independent of Hp phenotypes in Jordanians. Contrary to this, a population study by Stern et al. [22] indicated that diabetes is associated with the Hp1-1 phenotypes. Results from Adinotey et al. [15] presented investigation in Ghanaians and support the position that diabetes is associated with $\mathrm{Hp}$ phenotypes. Their finding confirmed what has been earlier on reported [16]. However, it contradicts the observations by Stern et al. [22].

Table 1. Clinical and Biochemical Indices of Non-diabetics and Diabetics

\begin{tabular}{|c|c|c|c|c|c|}
\hline \multirow{3}{*}{ Parameter } & \multicolumn{4}{|l|}{ Participants } & \multirow{3}{*}{ Ref. range } \\
\hline & \multicolumn{2}{|c|}{ Non-diabetics } & \multicolumn{2}{|c|}{ Diabetics } & \\
\hline & Male & Female & Male & Female & \\
\hline No. of persons & 11 & 39 & 12 & 38 & \\
\hline Age (years) & $41.18 \pm 9.00$ & $51.33 \pm 12.08$ & $61 \pm 8.76 b$ & $54.5 \pm 11.41$ & \\
\hline BMI (Kgm-2) & $27.42 \pm 5.04$ & $27.19 \pm 5.31$ & $25.18 \pm 4.83$ & $31.29 \pm 6.23^{\mathrm{c}}$ & $18.8-24.9$ \\
\hline Systolic (mmHg) & $118.90 \pm 10.48$ & $124.92 \pm 12.48$ & $138.00 \pm 27.15^{b}$ & $133.47 \pm 23.61^{\mathrm{a}}$ & $\leq 120$ \\
\hline Diastolic (mmHg) & $72.18 \pm 10.52$ & $72.41 \pm 11.16$ & $77.5 \pm 20.04$ & $79.5 \pm 13.40^{\mathrm{a}, \mathrm{c}}$ & $\leq 80$ \\
\hline FBG $(\mathrm{mM})$ & $4.73 \pm 0.40$ & $4.79 \pm 0.57$ & $9.19 \pm 4.41^{\mathrm{b}}$ & $7.26 \pm 3.10^{\mathrm{a}, \mathrm{c}}$ & $4.2-6.4$ \\
\hline
\end{tabular}

${ }^{\mathrm{a}}$ Significant difference $(\mathrm{p}<0.05)$ between diabetics and non-diabetics, ${ }^{\mathrm{b}}$ Significant difference $(\mathrm{p}<0.05)$ between diabetic males and non-diabetic males, ${ }^{\mathrm{c}}$ Significant difference $(\mathrm{p}<0.05)$ between diabetic females and non-diabetic females. 

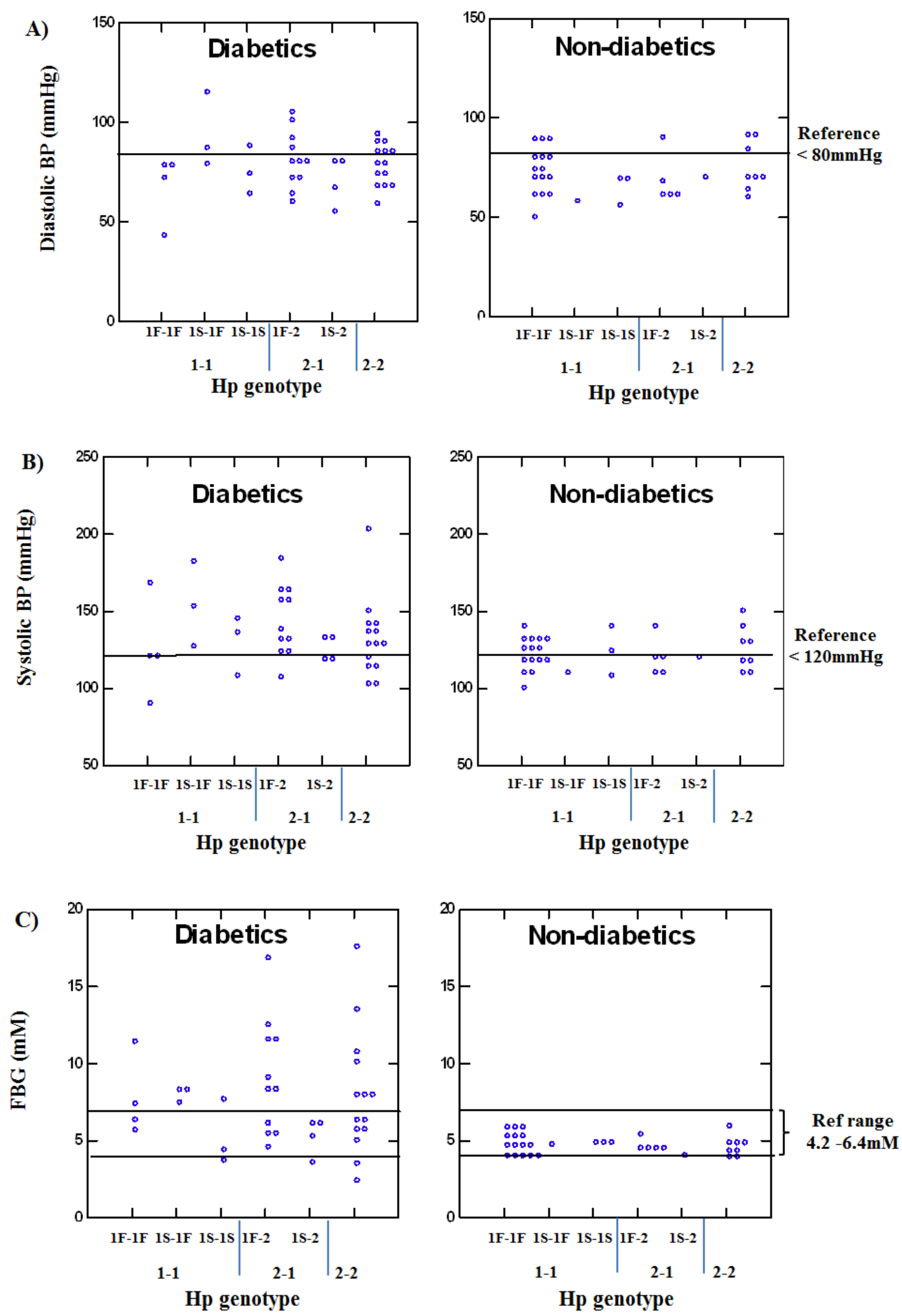

Figure 3. Dot density plots for the diabetics and non-diabetics (A) Hp genotype distribution and diastolic BPs (mmHg) (B) Hp genotype distribution and systolic BP (mmHg) (C) Hp genotype distribution and mean fasting blood glucose ( $F B G)(\mathrm{mM})$. (*outlier/s)

Results of this study confirm the work by Adinortey et al. [15]. There is a strong association between DM and Hp2-2 genotype followed by Hp2-1 (Hp1F-2 > Hp1S-2) with the least being Hp1-1 (Hp1F-1F, Hp1S-1F, Hp1S-1S). This supports the fact that Hp1-1 individuals show better protection against DM than Hp2-1 and Hp2-2 individuals. Hp2-2 exhibits hindrance effect leading to poor clearance of $\mathrm{Hb}$ in circulation [7].

It has been shown by several groups that the Hp2-2 polymorphism confers a $2-5$ fold increased risk for CVD 
compared to the Hp1-1 and Hp 2-1 genotype [2,23]. The underlying mechanism for the specific interaction between the Hp2-2 genotype and DM appears to be the result of its interaction with $\mathrm{Hb}$. In $\mathrm{DM}$ individuals, extravascular and intravascular hemolysis occur at higher rates compared to non-DM individuals, thus increasing the amount of $\mathrm{Hp}-\mathrm{Hb}$ molecules in plasma and tissues. $\mathrm{Hp}$ is considered an antioxidant due to its ability to scavenge $\mathrm{Hb}$ and prevent the initiation of radical chain reactions. However, this antioxidant activity varies greatly between the different $\mathrm{Hp}$ genotypes [24]. While the affinity for $\mathrm{Hb}$ is similar for Hp1-1 and Hp2-2 [5] the ability to seclude the hem-iron from its aqueous surrounding is greatly decreased in the latter. This disparity is further magnified by oxidation and glycation of $\mathrm{Hb}$, both common in DM [25].

\section{Conclusion}

A significant difference in the Hp genotype distribution between diabetics and non-diabetics was observed. Hp1-1 was the most frequent genotype among non-diabetics (58\%) whilst Hp2-2 (38\%) was most frequent genotype among diabetics. Non diabetics outnumbered the diabetics in the Hp $1 \mathrm{~F}-1 \mathrm{~F}$ group whereas diabetics outnumbered the non-diabetics in the Hp1S-1F, Hp2-1 and Hp2-2 groups. The risk of developing diabetes among people with $\mathrm{Hp} 2-2$ and Hp1S-1F genotypes were high and thus they can be used as markers for an individual developing DM.

\section{References}

[1] M. Polonovski, M.F. Jayle. Sur la preparation d'une nouvelle fraction des prote'ines plasmatiques, l'haptoglobine. Comptes Rendues des Se'ances de la Socie'te ' de Biologie, vol. 211, pp. 517-519, 1940.

[2] A. Roguin, W. Koch, A. Kastrati, D. Aronson, A. Schomiga, A.P. Levy, Haptoglobin genotype is predictive of major adverse cardiac events in the one year period after percutaneous transluminal coronary angioplasty in individuals with diabetes, Diabetes Care, vol. 26, pp. 2628-231, 2003.

[3] A.P. Levy, A. Roguin, I. Hochberg, P. Herer, S. Marsh, F.M. Nakhoul, K. Skorecki, Haptoglobin phenotypes and vascular complications in patient with diabetes, N Engl J Med, vol.343, pp. 343:969-970, 2000.

[4] J.W. Baynes, Role of oxidative stress in development of complications in diabetes, Diabetes, vol. 40, pp.405-412, 1991.

[5] R. Asleh, S. Marsh, M. Shilkrut, O. Binah, J. Guetta, F. Lejbkowicz, B. Enav, N. Shehadeh, Y. Kanter, O. Lache, O. Cohen, N.S. Levy, A.P. Levy, Genetically determined heterogeneity in hemoglobin scavenging and susceptibility to diabetic cardiovascular disease, Circ Res, vol. 92, pp.1193-1200, 2003

[6] D. Farbstein, A.P. Levy, The genetics of vascular complications in diabetes mellitus, Cardiol Clin, vol. 28(3), pp.477-96, 2010
[7] M.R. Langlois, J.R. Delangh, Biological and clinical significance of haptoglobin polymorphism in humans, Clin Chem, vol. 42, 1589-1600, 1996.

[8] R. Asleh, A.P. Levy, In vivo and in vitro studies establishing haptoglobin as a major susceptibility gene for diabetic vascular disease, Vasc Health Risk Manag, vol. 1(1), 19-28, 2005

[9] S.K. Das, S.C. Elbein, The genetic basis of type 2 diabetes, Cell Science, vol. 2, pp. 100-131, 2006.

[10] M.B. Adinortey, B.A. Gyan, J.P. Adjimani, P.E. Nyarko, A.K. Nyarko, Is there any relationship between haptoglobin phenotype and retinopathy among Ghanaian diabetics? J Ghana Sci Ass, vol. 11(2), 9-15, 2009.

[11] WHO, Definition, diagnosis and classification of diabetes mellitus and its complications. Part 1, Diagnosis and classification of diabetes mellitus. Geneva, World Health Organization, (WHO/NCD/NCS/99.2), 1999.

[12] Joint National Committee on Prevention, Detection, Evaluation, and Treatment of High Blood Pressure, The Sixth Report of the Joint National Committee on Prevention, Detection, Evaluation, and Treatment of High Blood Pressure (JNC VI). Arch Intern Med, vol. 157, pp. 2413-2446, 1997.

[13] F.J. Fowkes, H. Imrie, F. Migot-Nabias, P. Michon, A. Justice, P. Deloron, A.D. Lufty, K.P. Day, Association of haptoglobin levels with age, parasite density, and haptoglobin genotype in a malaria-endemic area of Gabon, Am J Trop Med Hyg, vol. 74(1), 26-30, 2006

[14] WHO, Global status report on noncommunicable diseases 2010. Geneva, World Health Organization, 2011.

[15] M.B. Adinortey, B.A. Gyan, J.P. Adjimani, P.E. Nyarko, C. Sarpong, F.Y. Tsikata, A.K. Nyarko, Haptoglobin polymorphism and association with complications in Ghanaian type 2 diabetic patients, Indian J Clin Biochem, vol. 26(4), pp. 366-372, 2011.

[16] I.K. Quaye, G. Ababio, A.G. Amoah, Haptoglobin 2-2 phenotype is a risk factor for type 2 diabetes in Ghana, $\mathrm{J}$ Atheroscler Thromb, vol. 13, 90-94, 2006.

[17] M.W. Knuiman, T.A. Welborn, V.J. McCann K.G. Stanton, I.J. Constable, Prevalence of diabetic complications in relation to risk factors, Diabetes, vol. 35, pp. 1332-1339, 1986.

[18] A. Nyarko, K. Adubofour, F. Ofei, J. Kpodonu, S. Owusu, Serum lipid and lipoprotein levels in Ghanaians with diabetes mellitus and hypertension, J Natl Med Assoc, vol. 89, pp. 191-196, 1997.

[19] S.H. Atkinson, T.W. Mwangi, S.M. Uyoga, E. Ogada, A.W. Macharia, K. Marsh, A.M. Prentice, T.N. Williams, The haptoglobin 2-2 genotype is associated with a reduced incidence of Plasmodium falciparum malaria in children on the coast of Kenya, Clin Infect Dis, vol. 44, pp. 802-809, 2007.

[20] I. Kasvosve, Z.A.R. Gomo, E. Mvundura, V.M. Moyo, T. Saungweme, H. Khumalo, V.R. Gordeuk, J.R. Boelaert, J.R. Delanghe, D. De Bacquer, I.T. Gangaidzo, Haptoglobin polymorphism and mortality in patients with tuberculosis Int J Tuberc Lung Dis, vol. 4, pp. 771-775, 2000.

[21] S. Awadallah, M. Hamad, A study of haptoglobin phenotypes in patients with chronic renal failure. Ann Clin Biochem, vol. 40(6), pp. 680-683, 2003. 
[22] M.P. Stern, R.E. Ferrell, M. Rosenthal, S.M. Haffner, H.P. Hazuda, Association between NIDDM, RH blood group, and haptoglobin phenotype. Results from the San Antonio Heart Study. Diabetes vol. 4, pp. 387-391, 1986.

[23] A.P. Levy, I. Hochberg, K. Jablonski, H.E. Resnick, E.T. Lee, L. Best, B.V. Howard; Strong Heart Study, Haptoglobin phenotype is an independent risk factor for cardiovascular disease in individuals with diabetes, The Strong Heart Study. J Am Coll Cardiol, vol. 40(11), pp.1984-1990, 2002.
[24] M. Melamed-Frank, O. Lache, B.I. Enav, T. Szafranek, N.S. Levy, R.M. Ricklis, A.P. Levy, Structure-function analysis of the antioxidant properties of haptoglobin. Blood, vol. 98(13), pp. 3693-3698, 2001.

[25] R. Asleh, J. Guetta, S. Kalet-Litman, R. Miller-Lotan, A.P. Levy, Haptoglobin genotype- and diabetes-dependent differences in iron-mediated oxidative stress in vitro and in vivo, Circ Res. vol. 96(4), pp. 435-441, 2005. 